\title{
A Composite Damage Tolerance Simulation Technique to Augment the Building Block Approach
}

\author{
Mark McElroy, ${ }^{1}$ \\ NASA Johnson Space Center, Houston, TX, 77058, USA \\ Mohammad Zanganeh, ${ }^{2}$ \\ Jacobs Technology at NASA Johnson Space Center, Houston, TX, 77058, USA \\ Matthew Galeano ${ }^{3}$ and Jeremy Jacobs ${ }^{4}$ \\ NASA Johnson Space Center, Houston, TX, 77058, USA
}

\begin{abstract}
Aerospace structures comprised of composite materials are traditionally certified empirically via the Building Block Approach (BBA). While this approach has been performed successfully in the past, it is expensive and time consuming. One means to improve the overall efficiency of composite structural certification is to reduce the cost of the $\mathrm{BAA}$ by eliminating the need for some tests by incorporating damage analysis tools. For an analysis to replace a given test, the tool must first be validated using other similar test data. The subject of this paper is a description of an analysis technique for simulating compression after damage strength of a solid laminate. The analysis technique is one that is practical for use in an applied engineering context due to efforts to minimize necessary computational resources and complexity of the model.
\end{abstract}

\section{Introduction}

Carbon-fiber reinforced polymer (CFRP) laminate materials are becoming increasingly common in aerospace structures. Often design requirements include a need for damage tolerance. The most widely accepted certification methodology to meet strength and damage tolerance requirements is use of the Building Block Approach (BBA) [1, 2]. The BBA consists of a test program beginning with basic material characterization, followed by more focused testing including damage tolerance, evaluation of structural features/elements, and concluding with full scale hardware tests under flight load conditions. The biggest drawback of this methodology is that it is very cost and time intensive. One opportunity for reducing this cost is incorporating damage simulation analyses that can reduce the number of required tests. This may be achieved by validating a modeling technique using tests performed to a level of confidence that the model can be applied in place of other similar tests that would have otherwise been required.

When considering damage tolerance, a common design driver for composite laminates is in-plane compression strength [3]. Therefore, the compression after impact (CAI) test is a common component in a typical BBA characterization effort. The CAI test is used to determine reduced compressive strength of a material system after impact damage has occurred. Prediction of this reduced strength using damage tolerance analysis tools [4-6] can potentially reduce the need for some of these tests provided that the modeling technique is validated using similar test data.

CAI testing was performed on a solid laminate carbon-fiber reinforced polymer composite material system as part of the BBA for the Orion spacecraft structural design and certification. Retroactively, a finite element analysis technique was developed using the Orion test data that could be used in future similar projects. The Orion BBA test data was used to help develop and correlate the modeling technique described in this paper, however, the model itself was never applied within the Orion program as suggested. Therefore, the modeling technique presented in this paper is best viewed as a proposed component of a future composite certification program. Use of the Orion context and Orion test data provides an excellent context for this demonstration of how damage simulation may be applied.

\footnotetext{
${ }^{1}$ Aerospace Engineer, Engineering Structures Division

${ }^{2}$ Senior Structural Engineer, Engineering Structures Division

${ }^{3}$ Materials Engineer, Engineering Structures Division

${ }^{4}$ Materials Engineer, Engineering Structures Division
} 


\section{Testing}

Compression after impact tests were performed as part of the Orion BBA for all of the composite material configurations (i.e., layups). While many configurations are used throughout the vehicle, testing and analysis of one solid laminate layup will be discussed in this paper. Test coupons consist of a woven IM7/977-3 CFRP material system. The layup consists of two $\left[+45^{\circ} / 0^{\circ} /-45^{\circ} / 90^{\circ}\right]_{2 s}$ laminates bonded to one another with a film adhesive layer (16-plies/adhesive/16-plies). Lamina properties for this material system that were used in the analyses described later in this paper are shown in Table 1. The adhesive layer was ignored in the analysis by treating it as if it were the same as any other ply interface in the layup. All material and strength properties are typical values. In addition to the properties listed in Table 1, fracture properties including critical energy release rate and a mixed-mode exponential parameter were used in the modeling, however, this data is not available for publication.

Table 1. Material properties.

\begin{tabular}{cccl}
\hline Property & Value & Units & Description \\
\hline$E_{11}$ & 10.19 & Msi & $0^{\circ}$ elastic modulus \\
$E_{22}$ & 10.77 & Msi & $90^{\circ}$ elastic modulus \\
$E_{33}$ & 1.29 & Msi & Transverse elastic modulus \\
$G_{12}$ & 0.40 & Msi & 12 shear modulus \\
$G_{13}=G_{23}$ & 0.43 & Msi & 13 and 23 shear modulus \\
$v_{12}$ & 0.044 & & Poisson's ratio \\
$v_{13}=v_{23}$ & 0.081 & & Poisson's ratio \\
& & & \\
$X^{T}$ & 149.11 & $\mathrm{ksi}$ & $0^{\circ}$ tensile strength \\
$X^{C}$ & 122.6 & $\mathrm{ksi}$ & $0^{\circ}$ compressive strength \\
$Y^{T}$ & 149.11 & $\mathrm{ksi}$ & $90^{\circ}$ tensile strength \\
$Y^{C}$ & 122.6 & $\mathrm{ksi}$ & $90^{\circ}$ compressive strength \\
$Z^{T}$ & 7.315 & $\mathrm{ksi}$ & Transverse tensile strength \\
$Z^{C}$ & 50.0 & $\mathrm{ksi}$ & Transverse compressive strength \\
$S^{12}$ & 17.2 & $\mathrm{ksi}$ & 12 shear strength \\
$S^{13}$ & 10.19 & $\mathrm{ksi}$ & 13 shear strength \\
$S^{23}$ & 10.19 & $\mathrm{ksi}$ & 23 shear strength
\end{tabular}

An overview of the CAI test setup that was used is shown in Figure 1. The CAI test procedure consisted first of impacting 4" x 6" test coupons in an impact fixture according to ASTM-D7136 (Standard Test Method for Measuring Damage Resistance for Fiber Reinforced Polymer Matrix Composite to a Drop Weight Impact Event) [7]. Various impact test parameters were evaluated in the Orion BBA, with boundary conditions selected to represent the relevant manufacturing threat environment (i.e., handling damage, tool drops, etc.). The model correlation exercise described in this paper is based on use of an energy level of $15 \mathrm{ft}$-lbs and an impactor tip geometry of $1 / 2$ " diameter semi-sphere. After impacting the coupons, flash infra-red thermography (Flash IR) non-destructive evaluation (NDE) was performed to ascertain the size of impact damage. Additionally, x-ray computed tomography (CT) and was performed to characterize the impact damage in greater detail. A representative view of NDE results of an impacted specimen (before compression loading) is shown in Figure 2. Note that different projected damage areas are observed using flash IR on the front and back sides of the specimen. After impact and NDE of the test coupons, the specimens were loaded in displacement controlled edgewise compression according to ASTM-D7137 (Standard Test Method for Compressive Residual Strength Properties of Damaged Polymer Matrix Composite Plates) [8] in a hydraulic-servo 100kip load frame. Strain data was collected from gauges bonded on both sides of each specimen. Applied force data was collected from the test frame load cell.

The goal of the test was to observe the critical compression strain of the material at ultimate failure and use this information to generate a structural allowable for the material with impact damage present. In the case of this test, the critical force is coincident with unstable onset of growth from the initial impact damage. The analysis results later in this paper use critical force as the correlation metric between the model and test. 


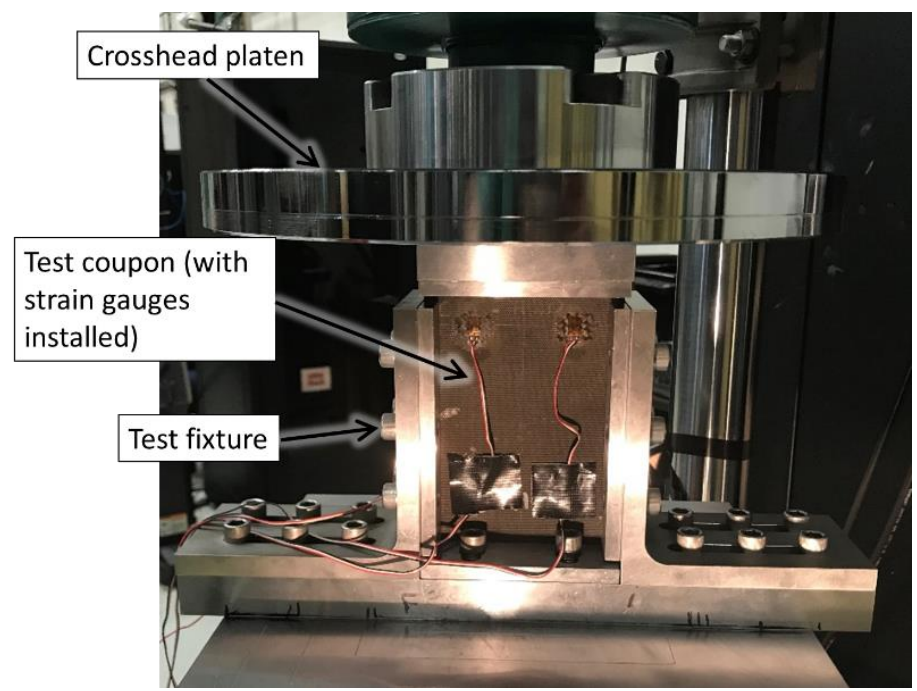

Figure 1. Compression after impact test setup

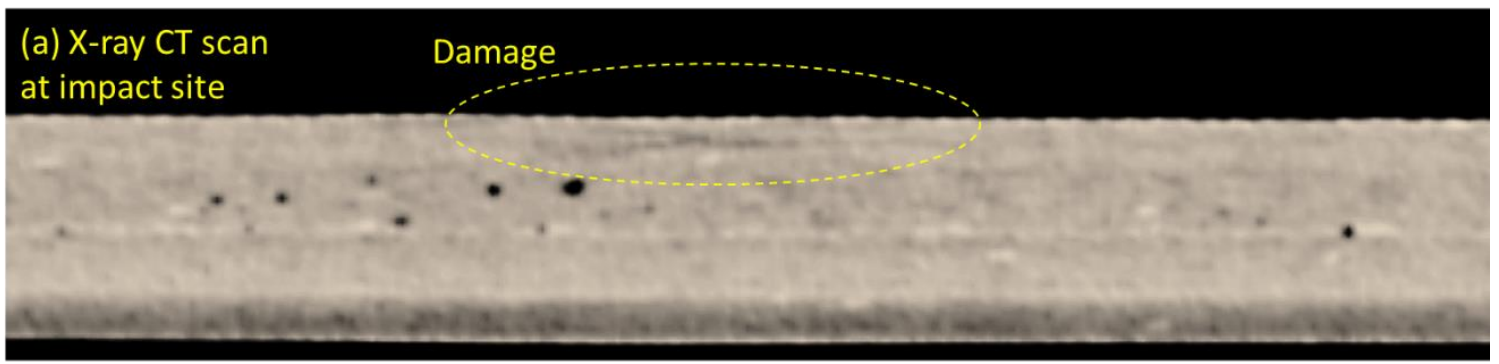

(b) Flash IR scan, impacted side

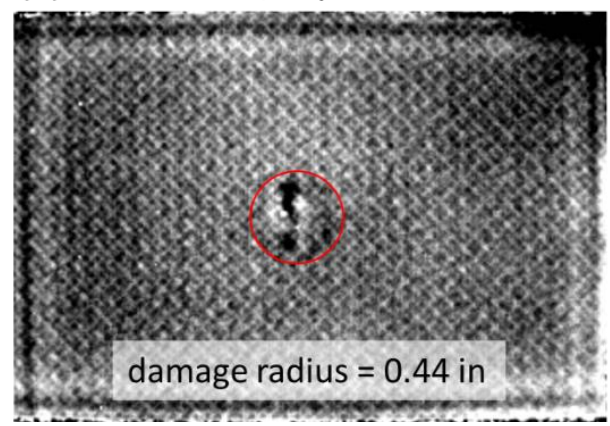

(b) Flash IR scan, back side

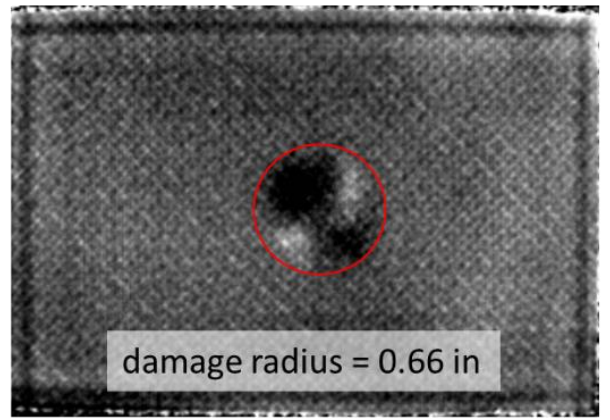

Figure 2. Representative Non-destructive Evaluation of Impact Damage. (a) X-ray CT scan at impact site and (b) Flash IR scan of impacted side (left) and back side (right)

\section{Model Definition}

Abaqus Standard 2017 was used to perform finite element simulations of the test. The following is an overview description of the finite element model and modeling technique used in this study. Sensitivity of certain model details on results is discussed in the following sections, however, the details of this baseline model description are constant throughout this paper.

\section{A. Model Description: Baseline}

An overview of the model is shown in Figure 3. The boundary conditions are shown in Figure 3 are representative of the test fixture shown in Figure 1. Compressive loading was applied as prescribed displacements at nodes along one boundary of the coupon. An Abaqus plug-in and Graphical User Interface, as shown in Figure 4, were developed 
to create and run all of the models in this study. The plug-in is noteworthy as it lends itself well to use of the analysis technique in an applied engineering context where quick modifications and rapid turnaround of analyses is useful. The plug-in functions such that the user enters a limited predesignated set of parameters and the model generation, solution and determination of damage initiation forces are executed automatically.

The modeling discussed in this paper does not include simulation of the actual impact event, therefore, it must contain a representation of the preexisting damage that was caused by the impact as an initial feature of the model definition. Preexisting impact damage is represented in this modeling technique as discrete circular delaminations in a three-dimensional mesh composed of continuum shell elements. The preexisting damage size corresponds to projected damage diameter observed in the flash IR NDE scans. If multiple preexisting delaminations are included in the model, the damage sizes of the outermost delaminations within the layup correspond to the different observed NDE sizes from each face of the coupon. Intermediate cracks are sized based on a linear interpolation through the layup thickness. If only one preexisting delamination is included in the model definition, its diameter corresponds to the maximum measured NDE damage size. A goal of this study was to confirm that this means of representing initial damage, while a simplification of the actual damage as seen in CT scan in Figure 2, is sufficient to achieve accurate predictions of critical force.

The mesh is subdivided according to the preexisting damage. Mesh regions adjacent to and in between delaminations may represent multiple plies using composite laminate theory. This technique of mesh division combined with the use of continuum shell element types allows for an efficient solution process. Mesh convergence studies were performed globally and local to the preexisting delamination fronts to verify that the size used would not produce significantly different results if refined further. Figure 5 shows the force-strain data correlation between the model and test data. This is useful as baseline observation that, damage predictions aside, the model is capturing the elastic response well.

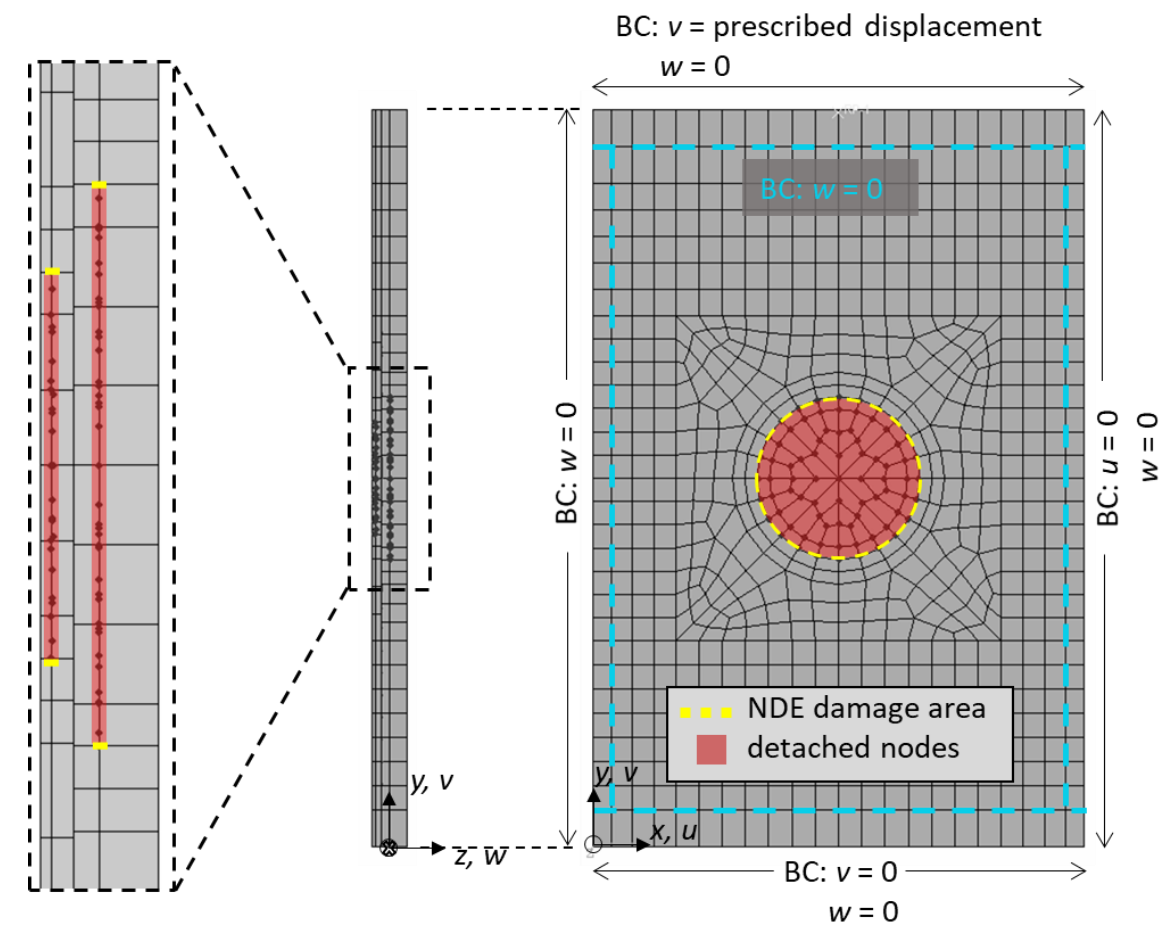

Figure 3. Finite element model overview (boundary conditions are applied such that displacement normal to the plate is restrained at all edges). 


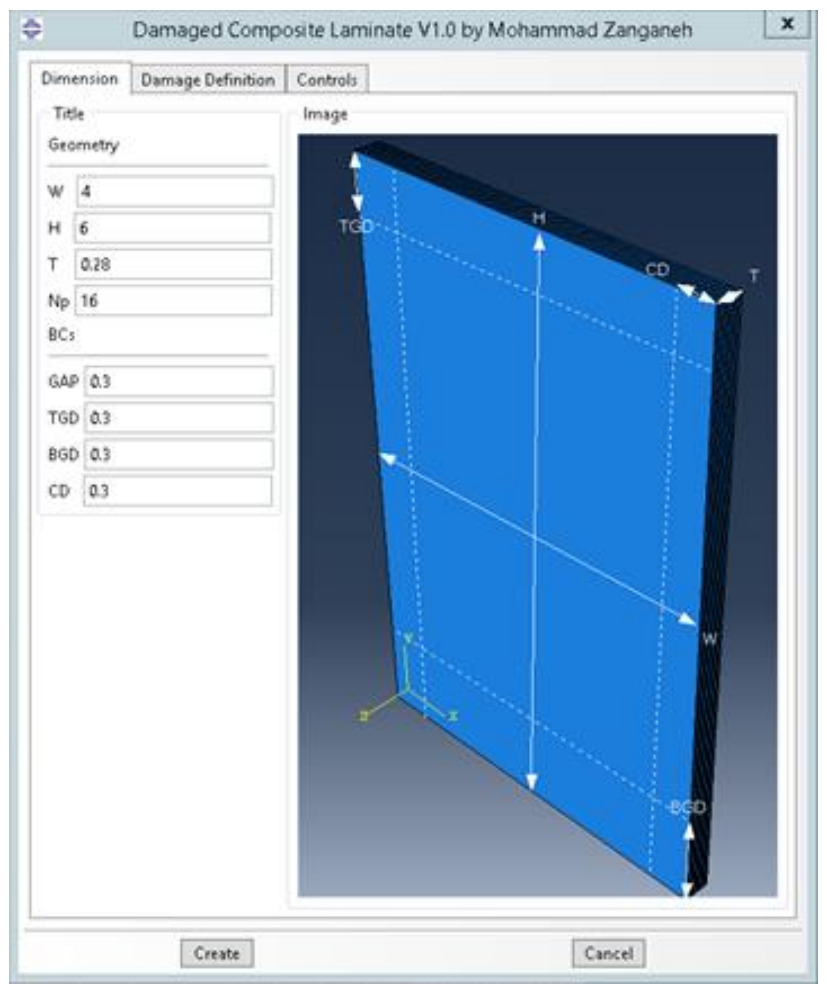

Figure 4. Snapshot of the Abaqus plug-in.

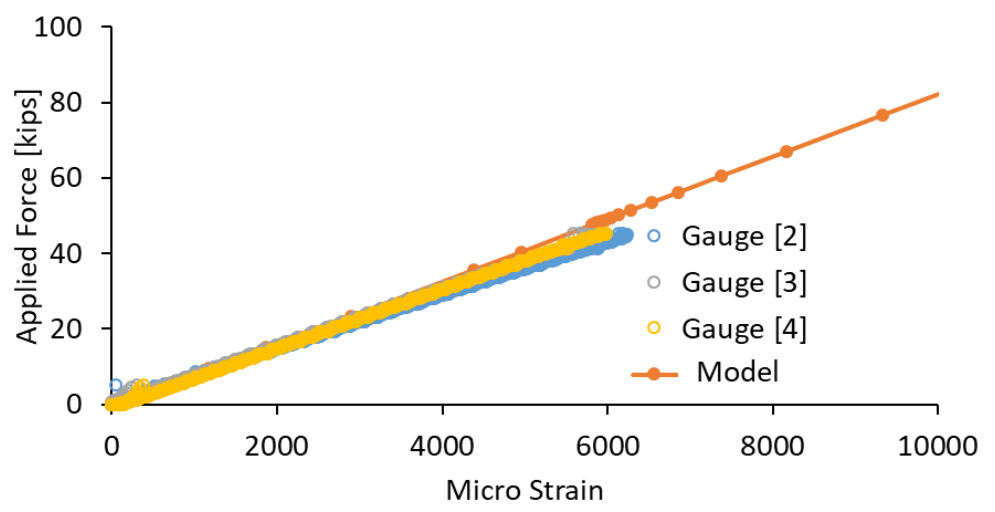

Figure 5. Force-strain data correlation between model and test (multiple strain gauges on front and back specimen).

\section{B. Model Description: Damage Prediction}

A major component of this investigation was determining a damage prediction technique that is accurate as well as computationally efficient. Recall, that the scope of this analysis is limited to prediction of the onset of growth from preexisting damage. There was no attempt to predict damage propagation as this behavior is not needed or observed in this particular BBA test. The numerical techniques to predict onset of growth from the preexisting damage were the Virtual Crack Closure Technique (VCCT) [9] and several first ply failure stress combination techniques including Hashin-Rotem [10], Tsai-Wu [10], Tsai-Hill [10], and Azzi-Tsai [10]. All damage onset numerical techniques were used in the form that is implemented in Abaqus 2017 (i.e., no user subroutines were used). Generally, the VCCT would be more adept at predicting damage if the critical failure model is interply (i.e., delamination) and the first-plyfailure stress criteria would be better at prediction damage if the failure mode if intraply (i.e., fiber failure). Mixed mode delamination onset was accounted for in VCCT using the Benzeggah-Kenane equation [11] to determine a critical energy release rate as a function of mode-mixity. 


\section{Parametric Study on Model Sensitivity and Abaqus Parameters}

An investigation was performed to study model sensitivity to various features and options within Abaqus. This investigation is summarized and presented here concluding with a modeling technique recommendation. The reader should also keep in mind that the purpose of these analyses was to develop a practical engineering tool and for this reason, simulation techniques requiring very fine meshes, multiple CPUs, or finely detailed representation of cracks were not considered. Sensitivity is posed in the context of correlating the critical force prediction in the model with that of the test. In the case of the $15 \mathrm{ft}-\mathrm{lb}$ impact, the test value to match is $43.9 \mathrm{kip}$. In the case of Hashin-Rotem, the value is shown that corresponds to the first failure index to exceed 1.0.

The main focus of the parametric study was to study details of how the preexisting damage should be defined in the mesh. The first sensitivity noticed was that of the type of contact algorithm definition used between delamination surfaces. Several contact algorithms exist for use in Abaqus. The contact algorithms considered in this study are listed in Figure 6. The effect on global response of the coupon in the model is illustrated qualitatively in Figure 6. The failure prediction is highly dependent on the global response. Case 1 and 4 are most representative of the global response observed in the test. Note that, in Abaqus 2017, when VCCT is enabled, the user has no control over the contact algorithm that is used.

\begin{tabular}{|c|c|c|c|c|}
\hline Case & VCCT Status & Contact Property option & Pressure Overclosure & Constraint reinforcement method \\
\hline 1 & On & VCCT Fracture Criterion & $\mathrm{N} / \mathrm{A}$ & $\mathrm{N} / \mathrm{A}$ \\
\hline 2 & Off & Normal Behavior & "Hard Contact" & Penalty \\
\hline 3 & Off & Normal Behavior & "Hard Contact" & Direct \\
\hline 4 & Off & Normal Behavior & "Hard Contact" & Default \\
\hline
\end{tabular}
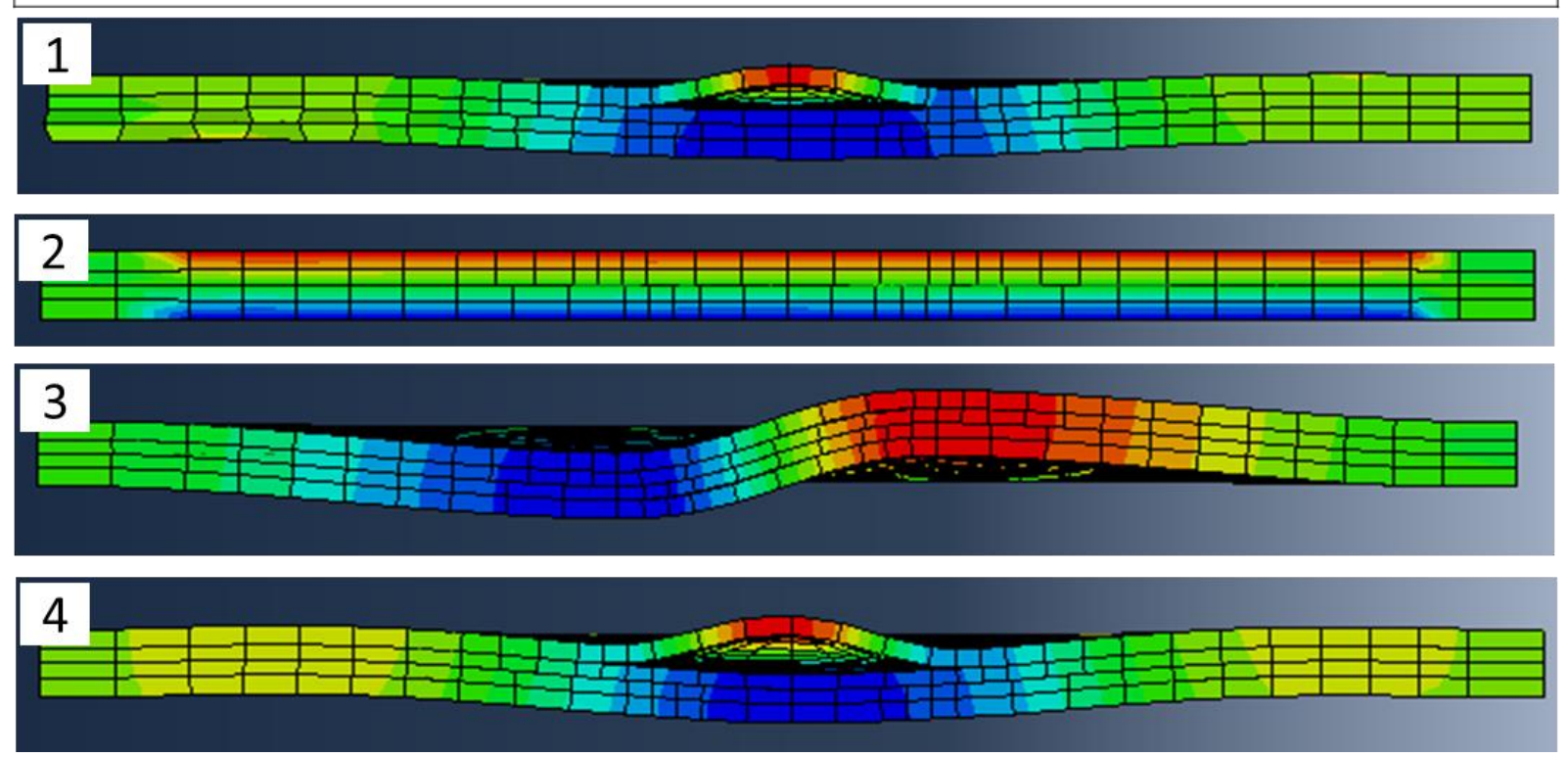

Figure 6. Sensitivity to contact algorithm.

Figure 7 illustrates other model parameters that were included in the sensitivity study: (a) starting depth of damage, (b) spread of two cracks, (c) eccentricity of two cracks, and (d) number of cracks. Results of the sensitivity study for the parameters illustrated in Figure 7 are shown in Figure 8. The experimentally observed critical force of 43.9 kip is identified as "target" in Figure 8. 

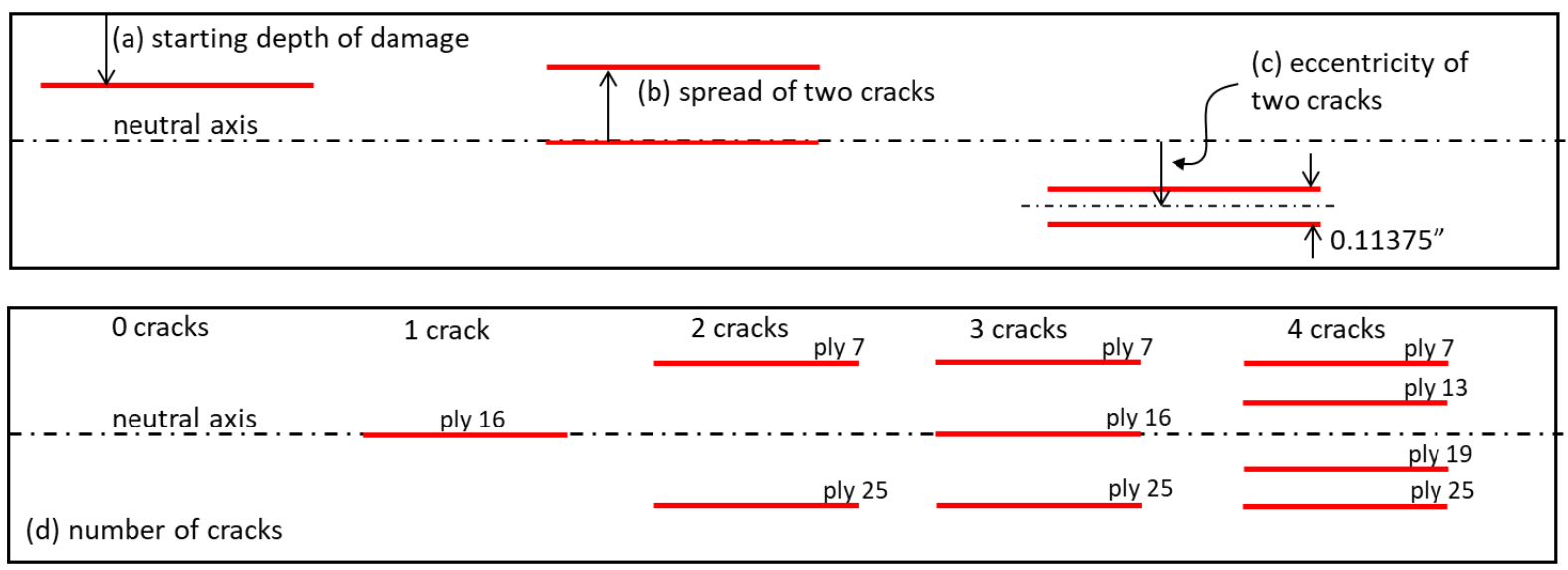

Figure 7. Definition of Parameters Included in Study.

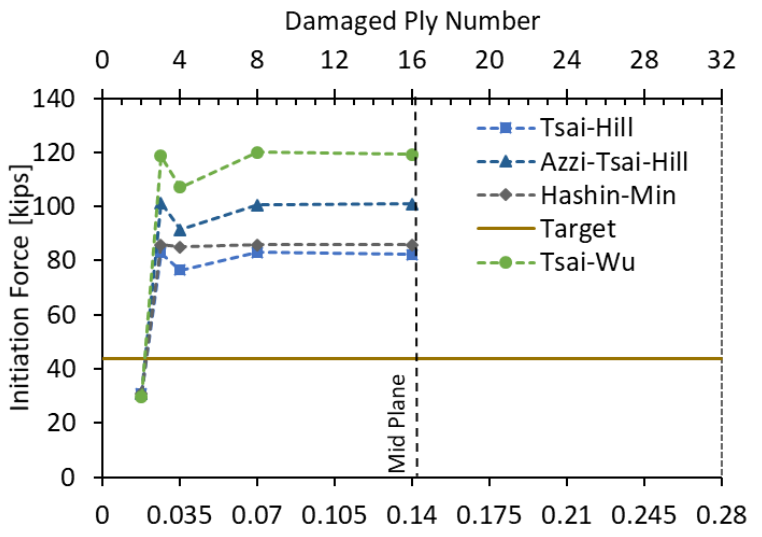

(a) Starting depth of damage [in]

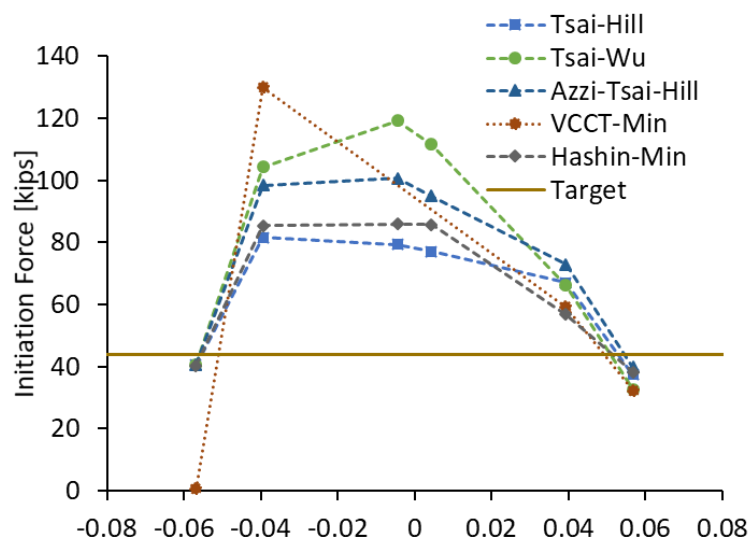

(c) Eccentricity [in]

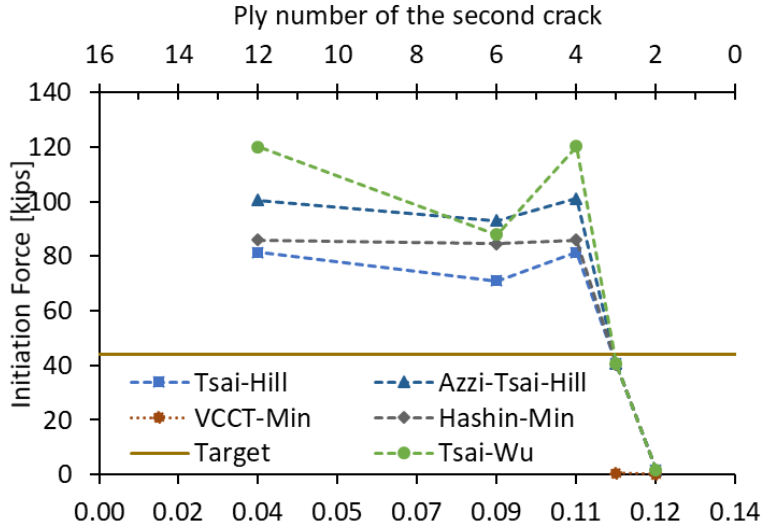

(b) Damage Spread [in]

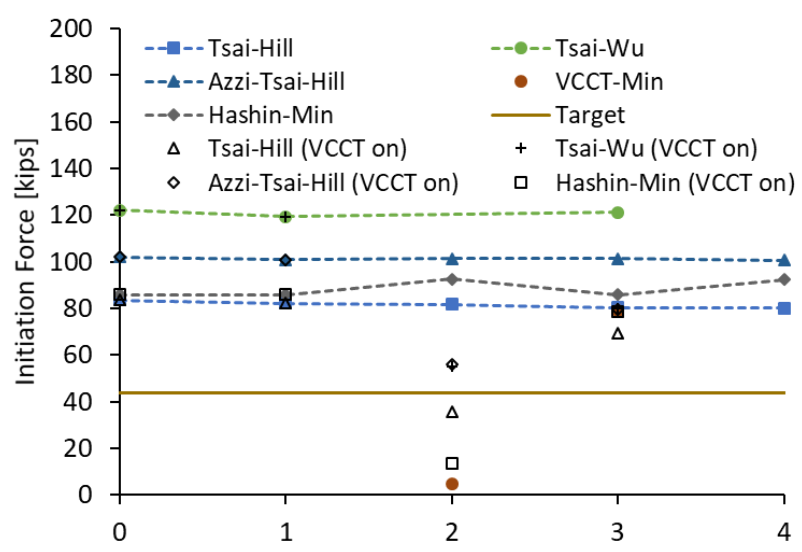

(d) Number of cracks [in]

Figure 8. Parameter sensitivity results.

Sensitivity of the model to starting depth of damage (i.e., ply interface) is shown in Figure 8a. If the starting depth of a crack is near the laminate surface, the critical force is under-predicted. If the crack is moved away from the laminate surface, a significant over prediction of the critical force is observed. The over prediction is essentially constant for each failure criteria aside from the case where the crack is near the surface. None of the model configurations in Figure 8a appear to be useful as a predictive tool. 
The sensitivity of the model to the distance of spread of two cracks is shown in Figure 8b. Generally, if VCCT is enabled, the model did not converge and in cases where it does, the critical force is close to zero. The stress criteria, while generating various levels of predictions, are insensitive to this parameter except for values greater than 0.105 ". Sensitivity in this range is thought to be a result of one of the cracks being positioned near the surface of the laminate.

The sensitivity of two preexisting delaminations to the eccentricity of their location (i.e., distance from the midplane) is shown in Figure 8c. The spread of the two delaminations is fixed at 0.11375". VCCT and stress-based results are sensitivity to this parameter. The results generated by the stress criteria appear correlate well with test data when the cracks are placed closer to the surface of the coupon. This matches the physical condition of the coupon itself as the impact damage is located near the surface. In the instances where good test correlation is observed in Figure 8c, all of the stress based criteria generate approximately the same prediction for critical force.

The sensitivity of the model to the number of cracks is shown in Figure 8d. The model is not sensitive to this parameter. However, if VCCT is activated, the stress based predictions change significantly. The VCCT prediction itself is well below the target value. This is likely due to the effect that the contact algorithm associated with VCCT has on the model (see Figure 6). Overall, enabling VCCT introduces convergence problems and has a high sensitivity to model parameters.

The ideal model configuration should be one that has parameters in a range where a high sensitivity is not observed and a good test correlation is observed. If this condition is met, the model will be more likely to produce reliable results while accommodating variations in the coupon such as NDE size or layup (as desired in order to use the model to replace similar otherwise needed tests). Based on the sensitivity study performed, the model condition that matches this requirement and correlates best with the test data is summarized as follows:

- Two preexisting delaminations spaced less than 0.11375 " apart

- Preexisting delaminations located near the impacted surface of the coupon

- Sizes of the two preexisting delaminations correspond to projected damage area from flash IR NDE of each side of the coupon

- Continuum shell elements, global mesh sized at approximately 0.2 in., maximum aspect ratio is 11.43

Use of VCCT to predict damage growth onset was not found to be reliable or useful in this application. The reasoning behind this is thought to be due to a high sensitivity to the contact algorithm plus the fact that onset of delamination does not appear to be the critical failure mode. The contact algorithm and contact behavior in general were found to influence prediction of delamination front energy release rate. Any of the stress based criteria appear to produce reasonable predictions in the ideal model configuration.

\section{Conclusion}

The overall result of this study is a test correlated damage simulation methodology for damage growth onset prediction in a CAI test. The demonstration in this paper is specific to one material system, one layup, and one preexisting damage state. Good correlation between the model and test data was observed. Furthermore, based on the parametric study that was performed, the model is believed to be configured in a state that is insensitive to slight variations in the test configuration. At the time this paper was written, further development of the model consists of evaluating the model's performance against other impact energies (i.e., other initial damage sizes). Because the work described in this paper was performed retroactively to the actual certification effort for the Orion vehicle, this work best serves as an example of what could be done in the future for a similar composite vehicle certification effort. The modeling technique developed in this paper is computationally efficient and implemented in an automated and easy to use Abaqus plug-in.

\section{Acknowledgements}

The authors would like to thank Dr. Mark Pankow and Tyler Goode of North Carolina State University for offering consulting on the modeling approach. 


\section{References}

[1] Composite Materials Handbook Volume 3, Polymer Matrix Composites: Materials, Usage, Design, and Analysis. SAE International, 2017.

[2] NASA-STD-5019 Fracture Control Requirements for Spaceflight Hardware. National Aeronautics and Space Administration, Washington, DC 20546-0001.

[3] Ratcliffe J, Jackson W, Schaff J. Compression strength prediction of impact damaged composite sandwich panels. In: Proceedings of the American Helicopter Society 60th Annual Forum, Baltimore, MD; 2004.

[4] Joglekar, S. Modeling and Simulation Strategies for Thickness Reinforced Composites. PhD Thesis, July 24, 2017.

[5] González, E. V., et al. "Simulating drop-weight impact and compression after impact tests on composite laminates using conventional shell finite elements." International Journal of Solids and Structures 144 (2018): 230-247.

[6] Tan, Wei, et al. "Predicting low velocity impact damage and Compression-After-Impact (CAI) behaviour of composite laminates." Composites Part A: Applied Science and Manufacturing 71 (2015): 212-226.

[7] ASTM D7136 / D7136M-15, Standard Test Method for Measuring the Damage Resistance of a Fiber-Reinforced Polymer Matrix Composite to a Drop-Weight Impact Event, ASTM International, West Conshohocken, PA, 2015, www.astm.org.

[8] ASTM D7137 / D7137M-17, Standard Test Method for Compressive Residual Strength Properties of Damaged Polymer Matrix Composite Plates, ASTM International, West Conshohocken, PA, 2017, www.astm.org.

[9] Krueger R. Virtual crack closure technique: history, approach, and applications. Appl Mech Rev 2004;57(2):109-43.

[10] Dassault systems. Abaqus 2017 analysis user manual; 2017.

[11] Benzeggagh M, Kenane M. Measurement of mixed-mode delamination fracture toughness of unidirectional glass/epoxy composites with mixedmode bending apparatus. Compos Sci Technol 1996;56(4):439-49. 